\title{
NOTICIA SOBRE EL ESTADO DE LA DOCUMENTACION EDUCATIVA EN COLOMBIA DURANTE EL PERIODO 1980 1986*
}

\author{
Gonzalo Alberto Rivera Martínez $^{\star \star}$
}

\section{PRESENTACION}

El Proyecto REDUC - Colombia con sede en el CIUP, empeñado en promover nuevas fases del procesamiento de la información educativa en el país, inició una investigación documental orientada a extraer de los Resúmenes Analíticos en Educación (RAE) publicados hasta el presente, una visión del estado de la documentación educativa en nuestro medio . Como primer paso en tal sentido, se elaboró un Informe de Avance (1) acerca de algunos aspectos descriptivos y evaluativos que se pudieron detectar de dicha documentación, según se desprende del material contenido en los seis volúmenes de RAE publicados entre 1983 -1986. Esta primera exploración fue igualmente útil para ajustar aún más las herramientas metodológicas con las que el proyecto REDUC aspira a pasar de la recopilación, organización y síntesis de la información al análisis de las posibilidades que ella presenta para la elaboración conceptual, particularmente mediante la producción de sucesivos Estados de Arte (2), tanto de orden general como particular, según la extensión temática abarcada y al nivel de lo teórico y/o metodológico. La noticia que se publica a continuación es una síntesis de las reflexiones contenidas en el mencionado Informe de Avance.

\section{ALGUNOS ASPECTOS METODOLOGICOS.}

El primer aspecto a resaltar del citado trabajo, es el de que se partió de los Resúmenes Analíticos en Educación (RAE). Esto significa que en lugar de estudiar documentos originales generalmente muy extensos, los investigadores pudieron aprovechar los RAE como instrumentos documentales que aceleraron y tecnificaron la tarea.

La celeridad se demostró en el hecho de que un periodo comparativamente corto se revisó el equivalente aproximado de 450 documentos que, de no haber existido los RAE, hubieran debido leerse en su integridad demandando una inversión de tiempo mucho mayor. Y la tecnificación que dichos RAE facilitaron, consistió en la visión integral que su lectura posibilitó de las características más relevantes de cada documento. Es decir, a través de los RAE los investigadores obtenían un primer nivel de procesamiento de información que les facilitaba detectar aquellos aspectos esenciales para su análisis y que no siempre en el documento original se encuentran expresados en forma explícita. Con ello quedó comprobado que para los futuros avances de la investigación documental, los RAE serán un instrumento capital para simplificar $y$, al mismo tiempo, volver más productivas las tareas que tal investigación demande.

\footnotetext{
*El trabajo base de esta "noticia" corresponde a la nota 1 del presente artículo.

** Master en Ciencia Política Universidad Javeriana. 
Con relación a jo anterior, la investigación permitió someter a prueba la consistencia y confiabilidad de las técnicas y conceptualizaciones que sustentan la elaboración de los RAE, así como de la manera en que se han venido produciendo hasta el momento.

Gracias pues, a esa visión sintética e integral resumida en los RAE, se pudieron definir 19 variables para describir las características más relevantes de los documentos originales sobre los que trabajan los RAE. Se establecieron dos instancias principales de descripción: la que podríamos llamar "Institucional" relativa a algunas características de las instituciones que participaron en la producción de la documentación analizada, y la que podríamos llamar "interna" al propio documento. En la primera instancia se observaba por ejemplo si la entidad que patrocinaba el trabajo era pública o privada, nacional o internacional, su sede, la clase de institución Universidad, Ministerio, Instituto Descentralizado, Fundación, Centro de Investigación y similares y la dependencia precisa donde se había originado la producción. Y en cuanto al documento propiamente dicho, además de tener en cuenta algunas características convencionales como la fecha de publicación, número de paginas y forma de presentación se analizaba si se trataba de un trabajo investigativo o no investigativo, el enfoque disciplinar predominante en su elaboración, el tipo de investigación de que se tratara (teórica, descriptiva o evaluativa), la clase de fuentes empleadas y las técnicas utilizadas. Además, aprovechando que los RAE incluyen las Ilamadas "palabras claves" o descriptores del Tesaurus en Educación empleados para clasificar los temas pertinentes a cada documento, se pudo observar qué tendencias existían en cuanto a distribución de las áreas temáticas más frecuentes dentro del universo documental explorado. Esta operación permitió apreciar cuan desatendidas se encuentran ciertas áreas consideradas como fundamentales en la investigación educativa.

La que hemos llamado segunda instancia de descripción (algunas características inmanentes a los documentos según lo consignado en los RAE permitió a los investigadores iniciar un proceso de sistematización de los trasfondos teóricos y metodológicos propios de esta forma de investigación documental, proceso que debe continuarse por tratarse de una labor pionera en el país.

\section{ALGUNAS CONCLUSIONES GENERALES}

\subsection{Situación de la documentación analizada en cuanto a su carácter investigativo.}

Es bastante notorio que si la documentación analizada se sometiera a un juicio severo desde las llamadas normas de la "comunidad científica", en su gran mayoría no podría scr considerada como material investigativo, por no alcanzar los niveles de rigor exigidos desde esa perspectiva. Se optó entonces, habida cuenta de las condiciones en que se desenvuelve la documentación educativa en nuestro medio, por aplicar criterios mucho más laxos con los cuales se clasificaron como "investigativos" un total de 354 (3) documentos resumidos en los RAE. Sin embargo, aún con esa flexibilidad no debe dejarse de señalar que predomina una debilidad general en los trabajos llamados "investigativos", la cual se refleja en los siguientes aspectos:

a) hay una desatención alarmante por la elaboración teórica. Trabajos de esta naturaleza son muy pocos en comparación con los evaluativos (4). No queremos subvalorar la importancia de estos últimos, pero conociendo la cantidad de problemas teóricos que denominan las preocupaciones sobre lo educativo y lo pedagógico (comenzando por su misma definición) no deja de ser impactan-te que ello no se refleje en el volumen de investigación producida. 
b) El interés por construir datos empíricos que sirvan como fuente de la investigación es igualmente minoritario frente a la utilización de fuentes secundarias. En Colombia ésto es muy preocupante dado el precario estado en que se encuentran las estadísticas que alimentan la reflexión sobre lo educativo. Pero hay un agravante más: al mismo tiempo que no hay preocupación por lo empírico, las técnicas dé análisis documental utilizadas son deficientes, no pasando por lo general, de mediocres revisiones bibliográficas y llegando sólo en contadísimos casos a los llamados Estados del Arte.

c) No deja tampoco de llamar la atención el hecho de que la investigación Acción o Participación, la Experimental y las denominadas técnicas etnográficas de investigación, representan dentro del volumen de documentación analizada un porcentaje ínfimo (5). En síntesis, ni la investigación convencional, ni las formas de investigación alternativa, presentan un balance positivo dentro del universo explorado.

\subsection{Una tendencia "institucionalizante" en la Documentación estudiada.}

Otra apreciación inquietante en torno de los documentos llevados a los RAE analizados y posiblemente muy relacionada con lo señalado en la sección anterior, es la del predominio del enfoque administrativo en el tratamiento de los temas. (6) No sólo pues domina la intención evaluativa, sino caen en el mundo de lo administrativo o de lo que algunos llamarían "economía de la educación" Este hecho obliga a pensar sobre qué tanto ese sesgo hacia el "saber administrativo" está obstaculizando el desarrollo del "saber pedagógico" que demanda sin contemplaciones la situación del proceso educativo en el país. Entre los investigadores responsables del Informe de Avance que aquí se sintetiza, existe el temor de que si se continúa por ese camino la documentación educativa puede caer en una tendencia de tipo "burocratizante" definitivamente nefasta para la investigación. Como es natural, contrarrestar tal tendencia demandaría aumentar los esfuerzos investigativos en el diagnóstico e interpretación del fenómeno y crear políticas para fortalecer otro frente de investigación con propósitos diferentes a los que son comunes en el llamado "saber administrativo". Desde todo punto de vista es muy grave que este último adquiera tal predominancia sin que paralelamente se desarrolle la producción teórica y se refinen los métodos y técnicas empleados en la investigación. Debe agregarse que quizás un elemento que contrarresta esa tendencia perjudicial, sería el de que continuara aumentando el recurso al enfoque pedagógico según aparece en la investigación (7).

\subsection{El "Centralismo" en la Documentación en Educación.}

Otra constatación que obliga a reflexionar es la de la hegemonía de la capital del país sobre el resto de las regiones en cuanto al volumen producido de documentación educativa. Esto es una nueva llamada de alarma para preocuparse acerca de cómo se están adelantando los esfuerzos por una descentralización del desarrollo cultural en un campo tan estratégico como lo es el de la Educación (8).

\subsection{La participación de la mujer en la producción de Documentación en Educación.}

Al comprobar el bajo porcentaje en que parece la mujer como autora de documentos sobre temas educativos es inevitable que surjan múltiples interrogantes, especialmente por tratarse de un campo en el que se conoce que la mujer ha ganado cada vez más mayor participación. ¿Por qué ésto no se corresponde a nivel de la producción documental? Otra pregunta que ameritaría sucesivas investigaciones y cuyas respuestas seguramente obligarían a modificar pautas institucionales, grupales e individuales en las conductas predominantes actualmente (9). 
3.5 Desequilibrio en el tratamiento de las áreas temáticas.

Hechas las primeras exploraciones en este campo se encontró que la educación preescolar, la educación técnica, la no formal y a permanente, así como la educación rural, comparativamente fueron en muy pocas veces objeto de preocupación de los documentos estudiados. Y la educación secundaria, aunque más frecuentemente, está bastante por debajo de la atención dedicada a la educación superior y la educación primaría (10)

Al observar la frecuencia en que se tratan algunos temas más específicos, se encontró que lo relacionado con docentes, tecnología educativa, fracaso escolar, currículo, investigación educacional, historia de la educación y la relación Comunicación y Educación, temas todos ellos capitales en la problemática educativa, tuvieron una pobre representación dentro del conjunto de la documentación sometida a estudio (11>. Y las menciones a materias aún más particulares como la lecto-escritura, las matemáticas, la alfabetización, las ciencias básicas, la geometría, medicina, ingeniería, sexología, filosofía, e historia, apenas si representaron un porcentaje ínfimo en la clasificación temática que se hizo (12) .Se advierte a los lectores interesados que en el Informe de Avance aparecen numerosas conclusiones más específicas que amplían el marco de análisis de las conclusiones generales aquí resumidas.

\section{LA PRESENTE INVESTIGACIQN Y OTRAS INVESTIGACIONES SIMILARES QUE LA ANTECEDIERON}

Con motivo de la realización del Tercer Seminario Nacional de Investigación en Educación, organizado por el CIUP, el año de 1986, se publicó un resumen de los antecedentes principales que precedieron dicho evento, a saber: el Primero y Segundo Seminario sobre Investigación en Educación realizados en 1970 y 1980 respectivamente; el documento titulado "El Fortalecimiento de la capacidad investigativa en Educación en Colombia: (1960-1981)' preparado por Clemencia Chiappe y Roberto Myers, y la investigación sobre el "Fenómeno de la investigación en Colombia (1960-1978)" adelantada por José Bernardo Toro y Agustín Lombana. (13)

Mirando en conjunto dichos antecedentes y confrontándolos con el trabajo investigativo que se comenta en esta ocasión se obtienen las conclusiones generales siguientes:

4.1 La situación de la investigación educativa diagnosticada durante el periodo 19701980 , no se ha modificado en forma significativa. Los resultados que preocuparon a los investigadores ocupados de analizar ese tema durante la década de los 70 , siguen sustancialmente iguales.

4.2 En concordancia con lo anterior, se observa que las recomendaciones formuladas en los documentos producidos con anterioridad al presente estudio, no fueron atendidas y por lo tanto no se han llenado los vacíos, ni corregido las deficiencias que ellas señalaban.

4.3 Algunas tendencias que se preveían como comportamiento de la investigación educativa durante la década de los 80 , no aparecen confirmadas a la luz de la documentación analizada del proyecto REDUC-Colombia.

Las anteriores conclusiones se pueden ilustrar con los siguientes hechos: 
a) En relación con las condiciones institucionales en que se produce la investigación educativa:

i. A este respecto, en el Primer Seminario realizado el año 1970, ya habían aparecido la preocupación sobre el peligro de la burocratización y la concentración de la investigación, por lo cual se propuso la necesidad de promover una política descentralista de la investigación en educación (14). Esto según se señaló en esta noticia, no ha sido tenido en cuenta y aún' persisten los signos de burocratización y centralización que 17 años atrás inquietaban a los participantes en ese Primer Seminario.

ii. En el estudio de Lombana y Toro dedicado a analizar la investigación educativa durante el periodo 1960-1978, se encontró que el sector público había dominado en la producción investigativa, llegando a un porcentaje del $67 \%$ del volumen de la producción (15) . Esta situación aún persiste pues, como se dijo atrás, el $60 \%$ de la documentación revisada en $\mathrm{cl}$ trabajo que se comenta proviene del sector público.

iii. En el estudio de Clemencia Chiappe y Roberto Myers señalaba que "la mayor parte de la investigación educativa en Colombia se ha realizado en Bogotá". Este hecho también hoy día permanece inmodificado (16).

b) En cuanto a la naturaleza de los estudios realizados:

En la reseña del trabajo de Chiappe y Myers se menciona el "predominio de los estudios descriptivos" en la documentación por ellos analizada (17) Esta tendencia en buena parte se confirma hoy día en cuanto que la investigación teórico -hermenéutica ha tenido muy poco desarrollo. Como se dijo en alguna sección anterior de esta noticia, ante todo la investigación se concentra en lo descriptivo y evaluativo. Valga la pena anotar que en el estudio de Toro y Lombana, ya se había registrado algo similar (18).

c) En cuanto a los enfoques en Chiappe y Myers apareció que los temas administrativos relacionados con al economía de la educación representaban un 55\% de la distribución temática (19). Se vio atrás que esta tendencia hacia lo administrativo quedó confirmada en el periodo 1980-1986 que cubre la documentación del proyecto REDUC-Colombia. De igual manera, en la reseña del trabajo de Toro y Lombana se dice que "desde el punto de vista temático, los aspectos administrativos y de control han sido la mayor preocupación de los investigadores" (20)

Con relación al mismo tema de los enfoques se hacía notar "una carencia de investigaciones educativas con enfoque antropológico, psicológico y político", señalamiento que se confirma en la investigación comentada en esta ocasión.

La diferencia notable entre la década del 70 y lo que va de la década del 80 , es la de que los enfoques pedagógico e interdisciplinario han tenido un repunte en cuanto a la frecuencia en su utilización. Reacuérdese que en el Segundo Seminario sobre Investigación en Educación celebrado el año de 1980, se anotaba que el número de investigaciones con enfoque pedagógico es muy escaso (22). Aparentemente este vacío ha tendido a llenarse en los años siguientes, reflejando el mayor interés que ha cobrado el estudio de aspectos delimitados del proceso de aprendizaje (23).

\section{d) En cuanto a las prioridades temáticas:}

Sobre esté punto puede afirmarse que ninguna recomendación de las sugeridas con apremio por los estudios que antecedieron la investigación comentada en esta noticia, ha sido tenida en cuenta. Revisando las temáticas que se propusieron en el Primer Seminario del año 1970 sobre las cuatro áreas del alumno, el docente, el currículo y las instituciones escolares, (24) se encuentra que prácticamente ninguno de los temas allí planteados como prioritarios para la investigación han recibido la atención que se 
recomendaba. Algo similar ocurre cuando se comparan los resultados actuales y lo que se había propuesto en el Segundo Seminario del año 1980. Allí se destacó la urgencia de emprender estudios sobre educación rural, sobre los docentes en todos sus aspectos, sobre la alfabetización, sobre la calidad de la educación, sobre la educación infantil, temas que siguen figurando con un bajo porcentaje de frecuencia de investigación (25). $Y$ si se consulta el trabajo de Toro y Lombana allí aparece el comentario de que 'la mayor parte de los estudios estaban orientados a analizar problemas de la educación formal y que la educación no-formal, el sector técnico-vocacional y la educación especial, parecían atraer muy poco la atención de los investigadores (26). Esta situación permanece la misma.

e) En cuanto a la difusión de la investigación educativa:

En el primer Seminario del año 1970 se evidenciaba la necesidad de implementar políticas que posibilitaran la divulgación de los resultados de la investigación en educación realizada en Colombia y en América Latina. Y al respecto se señalaba: "La debilidad en la difusión de los hallazgos de los distintos trabajos se origina y continúa produciendo un desfase entre el quehacer de los investigadores y las decisiones de las diferentes instancias que orientan la política educativa (27). Por su parte, el Segundo Seminario realizado diez años después destacó la "ausencia de políticas claras de difusión de resultados de las diferentes investigaciones", lo cual impedía "una repercusión directa en la postulación de políticas para el sector y una adecuada apropiación de sus hallazgos por parte de la comunidad educativa" (28). Y Toro y Lombana en su estudio recalcaban: "La escasa difusión que alcanzan los productos investigativos tiene un efecto negativo sobre la misma investigación: afecta directamente la creación de una sana tradición investigativa,.al dificultar la continuidad en el tratamiento de los problemas y la acumulación del saber" (29). Estos llamados de atención sobre el problema de la divulgación de la investigación educativa han incluido en otros estudios de los años que se analizan. A pesar de ésto, la situación actual sigue siendo sustancialmente la misma y ese objetivo de planeación y continuidad en la investigación aún está lejos de alcanzarse.

f) En cuanto a la participación en la investigación de distintos sectores y estamentos

En el Primer Seminario del año de 1970 se planteó que "en sus distintas etapas la investigación se enriquecería con la participación de las diversas agrupaciones profesionales, económicas, religiosas, políticas y sociales; de las asociaciones de padres de alumnos y por último de los propios jóvenes que han demostrado ser capaces de contribuir a la renovación de los objetivos, de los programas y de los métodos de enseñanza; es pues probable que la investigación adquiera todo su valor y su alcance si se asigna una función de creciente importancia a la colaboración de los intereses y al diálogo entre investigadores y docentes (30). Inútil recalcar que este propósito continúa siendo letra muerta en nuestro medio.

g) En cuanto al empleo de los métodos estadísticos:

Hacia finales de los años 70 se creyó que habría un aumento de la utilización de métodos estadísticos cada vez más sofisticados en la investigación educativa (31). Si bien es cierto que los métodos estadísticos se han vuelto un tanto mas frecuentes, este hecho no configura aún una tendencia importante, y se lo observa a la luz de la documentación del proyecto REDUC-Colombia, se encuentra que son pocos los estudios que podría clasificarse como "sofisticados". (32) 
h) Otras tendencias que no se han confirmado:

En el año de 1981 parecía que la investigación educativa comenzaría a darle mayor atención al tema de la calidad de la educación, que habría un mayor rendimiento conceptual, que aumentaría significativamente las investigaciones específicas a nivel micro y que la investigación etnográfica se fortalecería (33). Al mirar retrospectivamente estas proyecciones, el trabajo que se comenta en esta noticia no permite confirmarlas. Por el contrario, aparecería que ninguna de ellas se comportó en el sentido en que los indicadores de entonces parecían apuntar.

i) La investigación sobre la Investigación en Educación:

Al final del documento donde se reunieron los antecedentes al Tercer Seminario sobre Investigación en Educación (34) se constata que no abundan los trabajos que buscaran "una comprensión global del fenómeno" de la investigación educativa. Hoy puede afirmarse que se sigue careciendo de un esfuerzo institucional, capaz de canalizar recursos hacia un frente de investigación que ofrece múltiples posibilidades y que ayudaría a satisfacer parte de las numerosas necesidades que enfrenta la investigación educativa en nuestro medio. Esta tarea sigue siendo una actividad marginal de apenas algunos interesados que incursionan heroicamente en un campo prioritario para la política investigativa nacional.

Sobre este particular debe resaltarse que hoy día la existencia de las publicaciones de los RAE (Resúmenes Analíticos en Educación) que viene produciendo el Proyecto REDUC-Colombia, se convierte en un instrumento valiosísimo para toda la investigación documental, en cuanto que facilita la revisión rápida y técnica de un número de investigaciones, orientada a crear estados de la investigación educativa en el país. Quizás ésto en parte explique que la cobertura del trabajo que se presenta en esta noticia alcanzó a 450 documento, mientras que sumando el total de los que fueron objeto de estudio, por parte de Toro y Lombana (238 estudios) y de Chiappe y Myers (102) sólo se llega a 340 (35).

Es preocupante que este potencial de la base documental REDUC no pueda aprovecharse a causa de la insuficiencia de recursos disponibles.

\section{UNA PREGUNTA FINAL.}

Desde 1970 hasta el presente, la situación de la investigación educativa ha sido preocupante y sus problemas permanecen en lo fundamental insuperados.

Así lo detectaron los diversos estudios realizados a lo largo de esos años y así lo confirma el Informe de Avance que se reseña en este artículo.

Habrá alguna posibilidad, quedará alguna esperanza, para que dentro de 7 ó 10 años no se esté rindiendo el mismo informe de 1970, 1979, 1981 y 1987? Ustedes tienen la palabra.

(1) Veáse SUAREZ, Edgar; RIVERA, Gonzalo Alberto. "El fenómeno de la Investigación Educativa en Colombia 1980 -1986" Informe de Avance, CIUP, Bogotá, 1987 (copia a máquina). 
(2) Como ejemplo de estas posibilidades veáse RIVERA, Gonzalo Alberto. "Una Mirada al Fenómeno de la Investigación sobre Educación Rural en Colombia- Análisis sobre la Base Documental REDUC 1980-1985”. Bogotá, 1987 (copia a máquina).

(3) De los 500 RAE incluidos en los 6 volúmenes publicados hasta el presente, se analizaron 457 en total. Los otros 43 no se tomaron en cuenta por corresponder a documentos anteriores a 1980. En el análisis de la variable "naturaleza de la documentación" en las que se discriminaba la documentación según se considerara "investigativa", el total de respuestas que dio el procesamiento de datos fue 394, distribuidas en 354 investigativa (10\% aprox). La explicación de cómo se definieron las variables empleadas en la investigación se encuentran en el Informe de Avance, citado atrás el cual se extiende ampliamente en la presentación de la metodología empleada en la investigación.

(4) Al discriminar las distintas clases de documentos "investigativos" el total de respuestas y su distribución fue el siguiente:

$\begin{array}{lrc} & \mathrm{N} & \% \\ \text { Evaluativos } & 223 & 61,6 \\ \text { Teóricos } & 67 & 18,6 \\ \text { Descriptivos } & 58 & 16,1 \\ \text { Acción Experimental } & 12 & 3,3 \\ \text { TOTAL } & 360 & 99,3\end{array}$

(5) Como se ve en la nota anterior, sumando la investigación Acción y/o Participación y la Experimental, no alcanzan a un 4\%. La discriminación de las respuestas a que corresponde este porcentaje es la siguiente: 8 investigaciones Acción y/o Participación y 4 experimentales

(6) El análisis de la variable relativa al Enfoque Disciplinar dominante en los documentos estudiados arrojó los resultados siguientes:

$\begin{array}{lrr}\text { Enfoques } & \mathrm{N} & \% \\ \text { Administrativo } & 113 & 25,4 \\ \text { Pedagógico } & 96 & 21,5 \\ \text { Interdisciplinar } & 86 & 19,3 \\ \text { Sociológico } & 60 & 13,4 \\ \text { Psicológico } & 28 & 6,2 \\ \text { Histórico } & 26 & 5,8 \\ \text { Económico } & 20 & 4,4 \\ \text { Filosófico } & 10 & 2,2 \\ \text { Político } & 5 & 1,1 \\ \text { Antropológico } & 1 & 0,2 \\ \text { total } & 445 & 99,5\end{array}$


Debe agregarse que al analizar las entidades que mayoritariamente patrocinan la documentación educativa se encontró un predominio del sector público, así:

$\begin{array}{lrr}\text { Entidades } & \mathrm{N} & \\ \text { Públicas } & 226 & 60,0 \\ \text { Privadas } & 148 & 33,4 \\ \text { Mixtas } & 29 & 6,5 \\ \text { Total } & 443 & 99,9\end{array}$

Este hecho de alto peso del sector público (ya detectado en investigaciones hechas sobre la documentación de los años 70 según se menciona al final de la presente noticia) puede contribuir a aumentar el sesgo hacía lo ‘administratiyo" que se comenta.

(7) En comparación con los estudios sobre la década del 70, la documentación analizada recurrió comparativamente con mucha mayor frecuencia al enfoque pedagógico, lo cual es un dato importante. También hay que destacar el aumento de documentos realizados desde una perspectiva interdisciplinaria. Ambos resultados, el de lo pedagógico y el de lo interdisciplinario, permiten abrigar la esperanza de proyecciones que contrarrestan la tendencia "burocratizante" a la que se podría llegar si no hay a tiempo los correctivos necesarios.

(8) La participación de las ciudades en la producción de la documentación analizada fue la siguiente:

$\begin{array}{lrr}\text { Ciudades } & \mathrm{N} & \% \\ \text { Bogotá } & 298 & 72,1 \\ \text { Cali } & 34 & 8,2 \\ \text { Medellín } & 32 & 7,7 \\ \text { Manizales } & 10 & 2,4 \\ \text { Armenia } & 8 & 1,9 \\ \text { Neiva } & 7 & 1,6 \\ \text { Barranquilla } & 7 & 1,6 \\ \text { Bucaramanga } & 6 & 1,4 \\ \text { Cúcuta } & 5 & 1,2 \\ \text { Pereira } & 4 & 0,9 \\ \text { Florencia } & 2 & 0,4 \\ \text { Total } & 413 & 99,4\end{array}$

(9) La variable "sexo del autor" de la documentación estudiada se distribuyó así:

$\begin{array}{lrc}\text { Autor } & \mathrm{N} & \% \\ \text { Masculino } & 243 & 58,8 \\ \text { Femenino } & 87 & 21,0 \\ \text { Mixto (autor colectivo) } & 83 & 20,0 \\ \text { Total } & 413 & 99,8\end{array}$

(10) En la revisión de este aspecto la investigación alcanzó a analizar los cinco primeros volúmenes de los RAE publicados por REDUC. Se ubicaron aquellos documentos que tocaran con algunas de las modalidades de educación y los resultados se distribuyeron así: 


$\begin{array}{lrr}\text { Educación } & \mathrm{N} & \% \\ \text { Superior } & 76 & 28,6 \\ \text { Primaria } & 64 & 24,1 \\ \text { Secundaria } & 38 & 14,1 \\ \text { A distancia } & 26 & 9,8 \\ \text { Rural } & 18 & 6,7 \\ \text { Preescolar } & 16 & 6,0 \\ \text { Técnica } & 10 & 3,7 \\ \text { Media } & 7 & 2,6 \\ \text { No-formal } & 7 & 2,6 \\ \text { Permanente } & 3 & 1,1 \\ \text { total } & 265 & 99,3\end{array}$

(II) Sumando el total de las clasificaciones hechas de las mencionadas temáticas en los cinco primeros volúmenes de RAE, se obtuvo una cifra de 853 referencias. De ellas, tocantes expresamente a algún aspecto del tema de docentes solo hubo $65(7,6 \%)$, al tema de la investigación educacional 38 (4.4\%) al tema del currículo 29 (3,3\%), al tema del fracaso escolar $18(2,1 \%)$, al tema de la tecnología educativa $18(2,1)$, y al tema de la Historia de la Educación 26(3\%).

(12) Dentro del mismo universo de la nota anterior, el total de referencias expresas a materias especificas no suma sino $47(5,5 \%)$. De ellas la más frecuentes son las relativas a Matemáticas (12), Lenguaje (8), Lectura-escritura (6), Ciencias (6), Alfabetización (3) y Educación Física (3).

(13)Veáse "Cuadernos del Seminario-Antecedentes", Tercer Seminario Nacional de Investigación en Educación, CIUP, (sin fecha).

(14) Ibidem p 12. Se lee allí textualmente: "Como alternativa para evitar burocratización el Director del ICOLPE planteó la implementación de una política descentralista de la investigación en educación".

(15) Ibideni p.31

(16) j ibidetn p.33

(17) Ibidem p. 36

(18) Jbidern p. 49

(19) Ibidemp. 40. Dicho dato se obtuvo sumando los seis primeros items temáticos que aparecen en el cuadro publicado por Chiappe.

(20) ibídem p. 53

(21) Ibidem p. 35

(22) ibidem p. 19

(23) esta tendencia comenzó a delinearse hacia finales de la década del 70, si bien no adquirió después la fuerza que se le vaticinaba. Sin embargo, el relativo aumento 
que tuvo puede explicar en parte el repunte del empleo del enfoque pedagógico que se comentó al analizar la distribución de los enfoques disciplinares más utilizados. Veánse notas 6 y 7 de esta noticia.

(24) Ibídem p. 12-14

(25) Ibidem p. 17-19

(26) Ibidein p. 52-53

(27) Ibidem p. 15

(28) Ibidem p. 21

(29) Ibidem p. 54

(30) Ibidem p. 15-16

(31) Ibídem p. 39-40

(32) Del total de los 450 RAE analizados no llegaron a cinco los documentos investigativos que emplearan métodos estadísticos sofisticados.

(33) ibídem p. 42

(34) Ibidem p. $55-56$

(35) Ibídem p. 26 y 27. 